
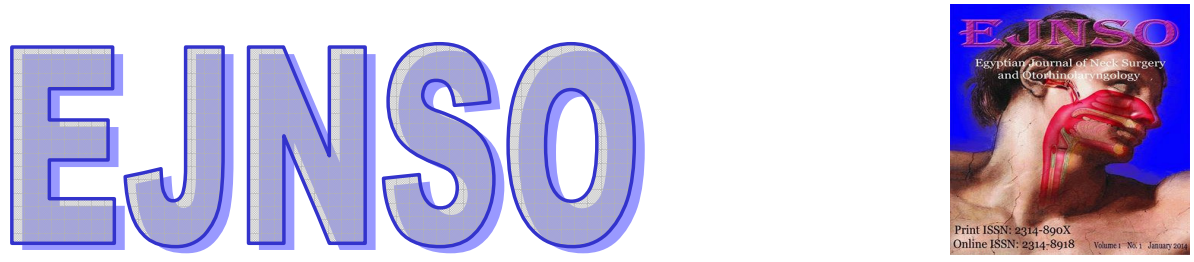

\title{
Supra-auricular Approach: A Simple- Recurrence Free- Technique for
} Pre-auricular Sinus

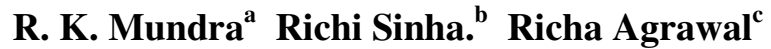 \\ Department of Otorhinolaryngology Head and Neck Surgery, MGM Medical College \& MY Hospitals, \\ Indore, Madhya Pradesh, India.
}

Received: October $20^{\text {th }}, 2013$.

Revised: November $3^{\text {rd }}, 2013$

Accepted:November $25^{\text {th }}, 2013$

KEYWORDS: congenital preauricular sinus, surgical treatment, supra-auricular approach, preauricular pit.

\begin{abstract}
Congenital preauricular sinus is a malformation of the preauricular soft tissues with an incidence ranging between 0.1 and $0.9 \%$ in Europe and the United States. It presents a high risk of recurrence when treated by a standard surgical technique (simple sinectomy); the incidence of which is reported to be between $19 \%$ and $40 \%$. The supra-auricular approach, proposed by Prasad et al. in 1990, is easier to perform and presents a lower recurrence risk. Personal experience was presented in the treatment of congenital preauricular sinus with the supra-auricular approach as first choice or in the case of primary as well as recurrence following previous standard surgery. This report justifies the use of supra-auricular approach as a very simple and effective procedure for the treatment of primary as well as recurrent preauricular sinus.
\end{abstract}

\section{INTRODUCTION}

The auricle develops from six mesenchymal proliferations surrounding the first pharyngeal cleft. These swellings fuse and gradually form the definitive auricle. As this fusion is a rather complicated process, developmental abnormalities may arise. A sinus results when one of the sulci between the auricular hillocks incompletely disappears. A preauricular sinus is a relatively common congenital abnormality. It consists of a blind-ending opening in the external ear, often located at or near the anterior crus of the helix. Preauricular sinus is more often unilateral, only occasionally are bilateral forms inherited. The right side appears to be more often involved and females more than males ${ }^{1,3}$. The embryological basis of this malformation is associated with a defect in the development of the first branchial arch during the sixth week of gestation.
Another hypothesis- less accredited- is that the sinus develops during embryonal auricular development from an isolated ectodermal folding ${ }^{2}$. From a clinical point of view, preauricular sinus is an occasional finding and most frequently appears like a small pit close to the anterior margin of the first ascending portion of the helix (Figure 1). In some cases, it is associated with a subcutaneous cyst in close proximity to the pit, related to the tragal cartilage and the anterior crus of the helix. The sinus course, in preauricular subcutaneous tissues, is not constant, often tortuous, with many ramifications, upwards and medially ${ }^{5}$. Topographically, the sinus is located more superficially than the temporalis fascia, laterally and superiorly from the parotid gland and facial nerve and the terminal cutaneous portion is very near and often adhering to, the cartilage of the first portion of the helix ${ }^{2}$. 


\section{CLINICAL FEATURES}

Most sinuses are clinically silent. The eventual appearance of symptoms, however not rare, is related to an infectious process. The most common pathogens causing infection are Staphylococcal species and, less frequently, Proteus, Streptococcus and Peptococcus 12. In this case, the tendency to recurrences requires surgical treatment. In the literature, associations have been described with renal or inner ear anomalies 6, 7 and there are a number of syndromes in which the preauricular sinus is only one of the clinical features 8,9 .

\section{MANAGEMENT}

Conservative management: Where no associated abnormalities are identified, and where the preauricular sinus is asymptomatic, there is consensus opinion that no further action is indicated. In the acute phase of infection, treatment comprises administration of appropriate antibiotics, and incision and drainage of an abscess if present.

Surgical management: In the symptomatic preauricular sinus exhibiting recurrent or persistent infection, opinion regarding optimal management varies. Latest evidence suggests definitive surgical treatment offering the most favorable outcome is by wide local excision of the sinus, as opposed to the previously preferred technique of simple sinectomy.

Other surgical techniques have been studied. The standard technique for extirpation of the sinus tract involves an incision around the sinus and subsequent dissection of the tract to the cyst near the helix. There is some agreement regarding surgical indication that is usually suggested after at least two subsequent infections Magnification employed during surgery, and opening, and following from the inside as well as outside, branching tracts of the sinus may further minimize the risk of recurrence.
Once infection occurs, the likelihood of recurrent acute exacerbations is high, and the sinus tract should be surgically removed. Surgery should take place once the infection has been treated with antibiotics and the inflammation has had time to subside. Controversy regarding indications for surgery exists. Some believe that the sinus tract should be surgically extirpated in patients who are asymptomatic because the onset of symptoms and subsequent infection cause scarring, which may lead to incomplete removal of the sinus tract and postoperative recurrences. The recurrence rate after surgery is $13-42 \%$ in smaller studies and $21 \%$ in one large study.

Most postoperative recurrences occur because of incomplete removal of the sinus tract. One way to prevent incomplete removal is to properly delineate the tract during surgery.

- Some surgeons cannulate the orifice and inject Methyline blue dye into the tract 3 days prior to surgery under sterile conditions

- Pre-operative sonographic imaging: preoperative sinograms aimed at grossly evaluating the sinus course ${ }^{2}$.

Each technical variant has limitations:

- The lacrimal probe trauma may cause a false course and it is unable to follow small ramifications.

- Methyline blue has a vital diffusion in tissues thus making correct identification of the smallest ramifications difficult.

- Fistulography needs experienced hands, far from acute infectious episodes, and offers only approximate indications regarding the sinus length and direction and no suggestion concerning its depth ${ }^{5}$.

A supposedly more successful technique is the supra-auricular approach (Figure 2), which unlike the former technique, does not allow for difficulties in properly identifying the entire tract. The supra-auricular approach extends the incision postauricularly. Once the temporalis fascia is identified, dissection of the tract begins. A portion of the auricular cartilage, which is attached to the tract, is also removed, decreasing the incidence of recurrence to 5\% (Figure 3). 
These issues are likely responsible for insufficient surgical radicality and consequently the high recurrence rate. Moreover, another aspect that appears to play an important role in the recurrence rate is the type of anesthesia; in our experience, in agreement with Yeo et al. ${ }^{14}$, surgery performed under local infiltrative anesthesia has a higher rate of recurrence than cases receiving general anesthesia. The reasons are probably related to patient's limited compliance with surgical maneuvers and to confounding factors that infiltration itself represents.

In 1990, Prasad et al. ${ }^{13}$, for the first time, described a new surgical approach defined supra-auricular approach which was based upon the theory that a fistula is, almost always, included in subcutaneous tissues between the temporalis fascia and perichondrium of the helix cartilage. Therefore, these Authors proposed that the elliptical incision of the standard technique be extended higher upward to the pre and supra-auricular temporal region. This allows better surgical vision without adverse aesthetic consequences.

Dissection proceeds identifying the temporalis fascia medially to the sinus area. It is only this fascia that represents a medial and deep limit of dissection that continues in a medium lateral direction until reaching the helix cartilage. At this level, dissection is made below the perichondral layer and, at the point of maximum adherence of the fistula; excision of a small portion of the cartilage is advisable.

The surgeon, during dissection, does not follow the fistula but, being aware of the space in which it develops, removes all subcutaneous tissue comprised between the temporalis fascia and the helix perichondrium in this tissue, the sinus is certainly present with its ramifications and the eventual cyst. The opening is then closed with a purse-string suture. This technique distends the tract and its extensions by its own secretion stained with Methyline blue.

During surgery, some surgeons use either a probe or an injection of Methyline blue dye for cannulation of the orifice. The most successful method is to use both modalities to delineate the entire tract.

Few papers related to this technique appeared in the Literature until 2001 when Lam et al. ${ }^{5}$ reported on a comparison between the two techniques, standard and supra-auricular, in two groups of patients (25 treated with the standard technique and 27 with the supraauricular) demonstrating how the latter presents a lower recurrence rate $(3.7 \% v s$. $32 \%$ with the standard technique) and concluding on the greater efficacy of supraauricular, compared with the standard technique in preauricular sinus treatment.

Aim of the present report is to contribute, with our limited experience, to increase the knowledge of the supraauricular approach, checking the real efficacy in recurrence risk reduction and to contribute to more widespread.

\section{PATIENTS AND METHOD}

There were 15 cases operated in our department of Otorhinolaryngology, MYH, INDORE. Ten of them were primary and 5 of them were recurrent cases.

TABLE 1: Patients, recurrence and follow-up times.

\begin{tabular}{|l|l|l|l|l|}
\hline Follow up & recurrence & Previous surgery & Primary/recurrent & No of patients \\
\hline$>2$ yrs & none & $\begin{array}{l}\text { None, only conservative } \\
\text { treatment given }\end{array}$ & primary & 10 \\
\hline$>1.5 y r s$ & none & Sinus excision & recurrent & 5 \\
\hline
\end{tabular}


Most of the patients came with acute tender preauricular swelling found out to be preauricular abscess due to infected preauricular sinus. All were first managed conservatively with or without incision and drainage and after 4-6 weeks they were operated upon. Meticulous follow up showed no recurrence.
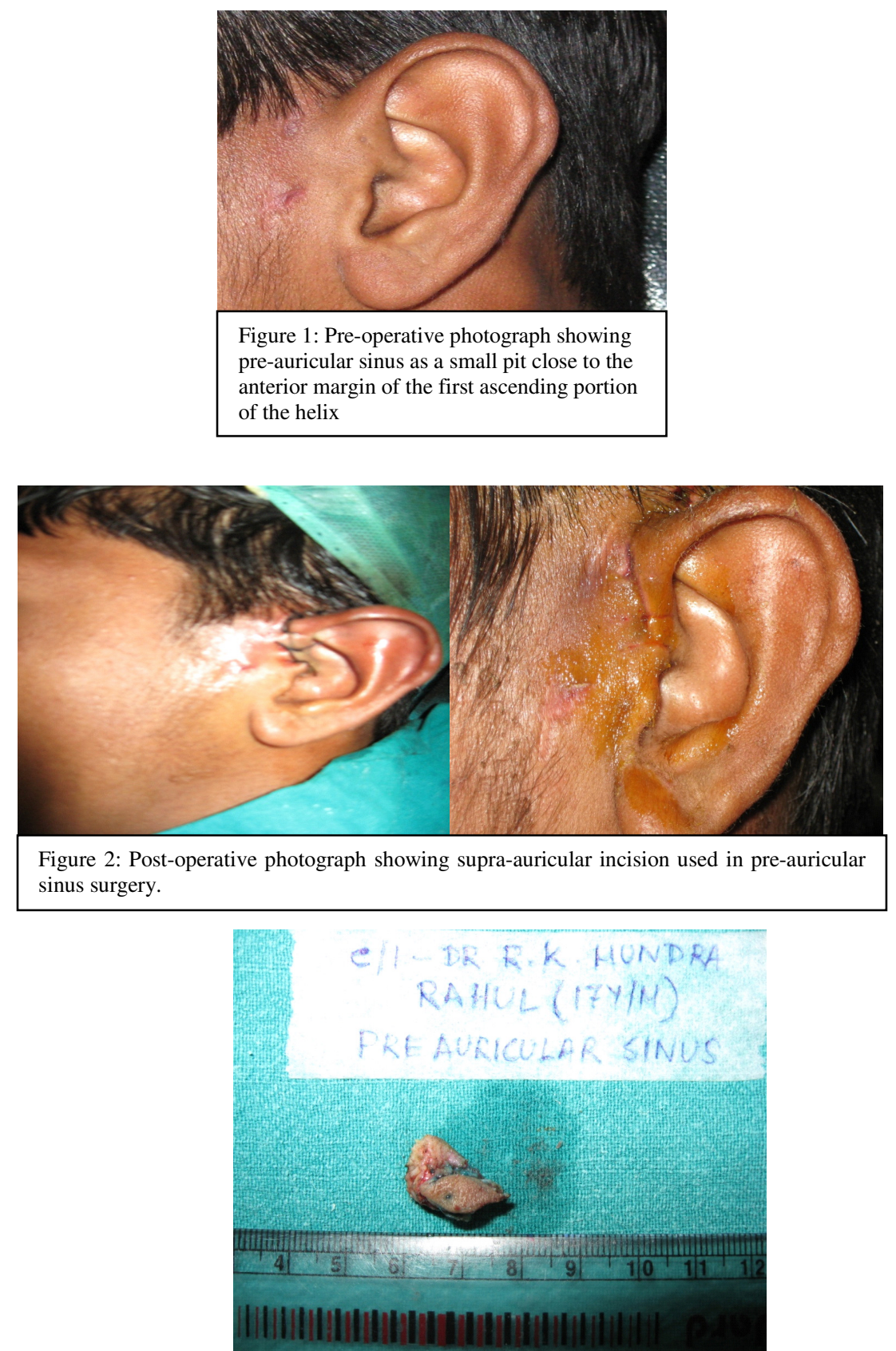

Figure 3: Photograph showing completely excised preauricular sinus by supra-auricular approach.

\section{DISCUSSION}

The real problem in the surgical treatment of preauricular sinus is the high recurrence rate following standard techniques due to the high variability of the sinus ramifications, particularly of the terminal ramifications which are diff cult for the surgeon to follow. Furthermore, infectious episodes, possibly with abscess, can produce scares that further alter the sinus route. The pre- and intraoperatory precautions, aimed to reduce this trend, are often not sufficient to guarantee absence of pathological recurrence.

Data in the literature confirm the limited efficacy of the standard technique and our own experience is in agreement with this conclusion. Therefore, based upon evidence from the literature and on the convincing rationale proposed for the supra-auricular technique, we decided to adopt this approach in all cases of preauricular sinus whether 
first surgical approach or recurrence following previous surgery.

Advantages of supraauricular approach:

- Simple

- Less time consuming

- Effective

- Less post-operative scar formation

- Can be done under LA

- Recurrence rate is negligible

Moreover, the supra-auricular approach offers fewer execution difficulties since it is not mandatory to isolate and to follow the sinus ramifications as in the standard technique but simply to identify a surgical plane, clearly defined and easy to dissect, such as the temporalis fascia.

\section{REFERENCES}

1. Scheinfeld NS, Silverberg NB, Weinberg JM, Nozad V. The preauricular sinus: a review of its clinical presentation, treatment, and associations. Pediatric Dermatol 2004; 21:191-6.

2 Tan T, Constantinides H, Mitchell TE. The preauricular sinus: a review of its etiology, clinical presentation and management. Int J Pediatric Otorhinolaryngol 2005; 69:1469-74.

3 Paulozzi LJ, Lary JM. Laterality patterns in infants with external birth defects. Teratology 1999; 60:265-71.

4 Nofsinger YC, Tom LWC, La Rossa D, Wetmore RF, Handler SD. Preauricular cysts and sinuses. Laryngoscope 1997; 107:883-7.
5 Lam HCW, Soo G, Wormald PJ, Van Hasselt CA. Excision of the preauricular sinus: a comparison of two surgical techniques. Laryngoscope 2001; 111:317-9.

6 Leung AKC, Robson WLM. Association of the preauricular sinuses and renal anomalies. Urology 1992; 40:259-61.

7 Wang RY, Earl DL, Ruder RO, Graham JM. Syndromic ear anomalies and renal ultrasound. Pediatrics 2001; 108:e32.

8 Huang XY, Tay GS, Wansaicheong GK, Low WK. Preauricular sinus: clinical course and associations. Arch Otolaryngol Head Neck Surg 2007; 133:65-8.

9 Smith PG, Dyches TJ, Loomis RA. Clinical aspects of the brachio-oto-renal syndrome. Otolaryngol Head Neck Surg 1984; 92:468-75.

10 Baatenburg de Jong R. A new surgical technique for treatment of preauricular sinus. Surgery 2005; 137:56770.

11 Currie AR, King WWK, Vlantis AC. Pitfalls in the management of preauricular sinuses. Br J Surg 1996; 83:1722-4.

12 Gur E, Yeung A, Al-Azzawi M. The excised preauricular sinus in 14 years of experience: is there a problem? Plast Reconstr Surg 1998; 102:1405-8.

13 Prasad S, Grundfast K, Milmore G. Management of congenital preauricular pit and sinus tract in children. Laryngoscope 1990; 100:320-1.

14 Yeo S, Jun B, Park S, Lee J, Song C, Chang K, et al. The preauricular sinus: factors contributing to recurrence after surgery. Am J Otolaryngol 2006:27:396-400. 